\title{
Comparison of the MODIS and VIIRS on-board SD and SDSM Performance
}

\author{
Amit Angal ${ }^{\mathrm{a}}$, Xiaoxiong Xiong ${ }^{\mathrm{b}}$, Ning Lei $^{\mathrm{a}}$, and Kevin Twedt ${ }^{\mathrm{a}}$ \\ ${ }^{a}$ Science Systems and Applications Inc., 10210 Greenbelt Rd, Lanham, MD 20706 \\ ${ }^{\mathrm{b}}$ Sciences and Exploration Directorate, NASA Goddard Space Flight Center,Greenbelt, MD \\ 20706
}

\begin{abstract}
The MODIS reflective solar bands (RSB) are calibrated on-orbit using a solar diffuser (SD) with its on-orbit degradation, or change in the bi-directional reflectance factor (BRF) monitored using a solar diffuser stability monitor (SDSM). By performing alternate observations of direct sunlight via an attenuation screen and of sunlight reflected diffusely off the SD, the SDSM monitors the on-orbit degradation of the SD. The MODIS SDSM has 9 detectors, covering wavelengths from 0.41 to $0.94 \mu \mathrm{m}$. Both Terra and Aqua MODIS instruments have successfully operated for more than 16 years on-orbit, with the SD experiencing significant degradation at the shortest wavelength (about $50 \%$ for Terra MODIS and about $20 \%$ for Aqua MODIS at $0.41 \mu \mathrm{m}$ ). The first VIIRS instrument on the Suomi NPP spacecraft was launched in October, 2011 and the follow-on instrument was launched in November, 2017 on the JPSS-1 spacecraft (now NOAA-20). Both the VIIRS instruments carry a MODIS-like SD and SDSM system with an improved design based on the lessons learned from MODIS. Unlike MODIS, the VIIRS SDSM collects data using 8 detectors covering a similar wavelength range as MODIS. A similar wavelength dependent SD degradation pattern is also observed in both VIIRS instruments. This paper provides a comparison of the on-orbit performance of the four instruments in terms of the on-orbit changes in the SDSM detector responses and on-orbit degradations of their SDs. The NOAA-20 VIIRS instrument is still in its first year of operation and hence the early performance of the Terra and Aqua MODIS and SNPP VIIRS is discussed to provide a perspective comparison.
\end{abstract}

Keywords: SDSM, SD, Degradation, NOAA20, VIIRS, MODIS

\section{INTRODUCTION}

The MODIS instruments, on the Terra and Aqua platform, have a full complement of on-board calibrators to provide inflight radiometric, spatial, and spectral characterization. The on-board calibrators include a solar diffuser (SD), a solar diffuser stability monitor (SDSM), a blackbody (BB) and the spectro-radiometric calibration assembly (SRCA). ${ }^{1}$ Measurements of the $\mathrm{SD}$, with its on-orbit bi-directional reflectance factor (BRF) change tracked using the SDSM, provide an anchor point to facilitate the calibration of the reflective solar bands (RSB). Visible Infrared Imaging Radiometer Suite (VIIRS) is a follow-on MODIS instrument that has similar capabilities to perform the RSB calibration. ${ }^{2}$ The VIIRS instruments were built by the same instrument vendor as MODIS and have 22 spectral bands: 14 RSB, 7 thermal emissive bands (TEB), and 1 day-night band (DNB). Although the wavelength range of the RSB is essentially the same, MODIS has 20 reflective solar bands, with additional spectral bands in the 0.90 to $0.94 \mu \mathrm{m}$ range.

As expected, some of the lessons learned from the MODIS design have led to an improved SD and SDSM calibration mechanism for Suomi NPP VIIRS, launched in October, 2011. Since launch in November, 2017, the follow-on VIIRS instrument onboard the NOAA-20 (N20) spacecraft has been operating successfully and collecting useful data to support its on-orbit calibration and generation of various science products. As expected, it applies a similar SD and SDSM based calibration mechanism. The performance of the MODIS SD and SDSM has been extensively reported in previous work. The focus of this paper is the early on-orbit performance

Further author information: (Send correspondence to Amit Angal.)

E-mail: amit.a.angal@nasa.gov 
comparison of N20 VIIRS relative to SNPP VIIRS, Terra MODIS and Aqua MODIS for the first 180 days of operation. Also compared is the long-term (6.5 years) performance of SNPP VIIRS with Terra and Aqua MODIS on a similar timescale. The SD degradation for the four instruments is computed as a function of solar exposure so as to provide a common baseline for comparison. Also discussed in detail is the performance of the SDSM detectors.

Although the SD and SDSM calibration mechanism is common to MODIS and VIIRS instruments, instrument specific design and operational configurations need to be considered for a better understanding of their on-orbit performance.The MODIS SDSM tracks the SD degradation for wavelengths less than $1 \mu \mathrm{m}$ using 9 detectors whereas VIIRS SDSM performs this activity via 8 detectors, each with a corresponding set of bandpass filters. ${ }^{3,4}$ The data in SDSM is obtained from three view positions: 1) Attenuated sun view; 2) Illuminated SD view (with SD screen in place for VIIRS and optional configuration for MODIS); 3) dark view to measure the background signal. A common behavior across the four instruments is the greater degradation of the SD at shorter wavelengths and greater response degradation of the SDSM detectors at longer wavelengths. At the time of this writing, Terra MODIS SD has experienced most degradation of around $50 \%$ at $0.41 \mu \mathrm{m}$, compared with $20 \%$ for Aqua MODIS and $40 \%$ for SNPP VIIRS. At $0.41 \mu \mathrm{m}$, the N20 VIIRS has shown a SD degradation of about $9 \%$ in the first 180 days of operation, which is better performance (less degradation) compared to the SNPP SD on a similar timescale.

\section{SDSM SYSTEM AND ON-ORBIT OPERATION}

The SDSM consists of a spherical integrating source (SIS) with a single input aperture and nine (8 in the case of VIIRS) filtered detectors. Each filter has a narrow spectral bandpass so that change in SD reflectance is effectively monitored at discrete wavelengths from $0.4 \mu \mathrm{m}$ to $1.0 \mu \mathrm{m}$. A three-position fold mirror enables the SDSM detectors to sequentially view the direct sunlight, dark signal, and the illuminated SD. The direct sunlight is attenuated via a $1.44 \%$ transmitting screen ( $0.1 \%$ attenuation in the case of VIIRS) to keep the radiance within the dynamic range of the SDSM's detectors. The mapping of the MODIS and VIIRS detectors to wavelengths is shown in Table I. It can be noted that MODIS SDSM detector $8(0.904 \mu \mathrm{m})$ is absent from VIIRS. The $0.904 \mu \mathrm{m}$ detector maps to MODIS band 17 which has a similar wavelength, but is not present in either VIIRS instrument. The first seven VIIRS SDSM detectors are filtered to measure the degradation of the same wavelength centers as the bands M1-M7.

Table 1. MODIS and VIIRS SDSM detectors and wavelengths $(\mu \mathrm{m})$

\begin{tabular}{|c|c|c|c|c|c|c|c|c|c|}
\hline SDSM Detector & D1 & D2 & D3 & D4 & D5 & D6 & D7 & D8 & D9 \\
\hline MODIS & 0.412 & 0.466 & 0.530 & 0.554 & 0.646 & 0.747 & 0.857 & 0.904 & 0.936 \\
\hline VIIRS & 0.412 & 0.445 & 0.488 & 0.555 & 0.672 & 0.746 & 0.865 & 0.926 & \\
\hline
\end{tabular}

The MODIS and VIIRS SDSM have been operated regularly via command uploads to the spacecraft. Table II provides a summary of the MODIS and VIIRS SDSM operation frequencies throughout the mission. During the early on-orbit checkout stages, the SDSM was operated more frequently (per-orbit to daily basis) to accurately characterize the initial degradation of the SD. The frequency of operation is gradually reduced as the missions progress in an attempt to prolong the operability of the SDSM beyond its design life. In absence of SDSM operation, the MODIS SD door is closed to prevent the SD panel from solar exposure. At present, a typical collect for Aqua MODIS includes an orbit of close calibration (SD door open and SD screen deployed), followed by an orbit of open calibration (SD door open and SD screen up). A similar sequence of operation was also followed in the first four years of Terra MODIS operation, until an anomaly with the SD door forced it to be left permanently open with the SD screen deployed. As currently implemented, the time-interval between the turn-on and turn-off of the SDSM is about 9 minutes for both MODIS instruments. In the case of VIIRS, there is no SD door and a solar attenuation screen is permanently in place in front of the SD panel. The duration of the SDSM collect is comparatively shorter, approximately 5 minutes for SNPP VIIRS and 3.25 minutes for N20 VIIRS. Normally, the SDSM collects data using alternate views of the SD and Sun. However, in the case 
of MODIS, it has also been operated in a fixed mode where it views the SD for a prolonged duration during the open calibration and the Sun during the close calibration.

Table 2. MODIS and VIIRS On-orbit SDSM Calibration Frequencies

\begin{tabular}{|c|c|c|c|}
\hline & Start Date & End Date & Calibration Frequency \\
\hline Terra MODIS & $02 / 24 / 2000$ & $03 / 21 / 2000$ & Daily (open + close, alt mode) \\
& $03 / 24 / 2000$ & $01 / 16 / 2002$ & Weekly (open + close, alt mode) \\
& $01 / 30 / 2002$ & $05 / 06 / 2003$ & Bi-weekly (open + close, fix mode) \\
& $07 / 02 / 2003$ & $02 / 17 / 2004$ & Weekly (close, alt mode) \\
& $03 / 02 / 2004$ & $02 / 03 / 2009$ & Bi-weekly (close, alt mode) \\
& $02 / 24 / 2009$ & Present & Tri-weekly (close, alt mode) \\
\hline Aqua MODIS & $06 / 13 / 2002$ & $06 / 30 / 2002$ & Weekly (open + close, alt mode) \\
& $07 / 07 / 2002$ & $12 / 26 / 2005$ & Bi-weekly (open + close, alt mode) \\
& $01 / 09 / 2006$ & $04 / 28 / 2008$ & Tri-weekly (open + close, alt mode) \\
& $05 / 20 / 2008$ & Present & Tri-weekly (close), Every 6 weeks (open), alt mode \\
\hline SNPP VIIRS & $11 / 08 / 2011$ & $2 / 28 / 2012$ & Once per-orbit \\
& $2 / 29 / 2012$ & $5 / 16 / 2014$ & Once per-day \\
& $5 / 16 / 2014$ & Present & Three times/week \\
\hline N20 VIIRS & $11 / 30 / 2017$ & $12 / 14 / 2017$ & Once per-orbit \\
& $12 / 14 / 2017$ & $01 / 06 / 2018$ & Once every other orbit \\
& $1 / 6 / 2018$ & Present & Once per-day \\
\hline
\end{tabular}

\section{ESTIMATION OF SD DEGRADATION}

A time-series of the ratio of the SDSM SD view response to its Sun view responses is used to determine the on-orbit degradation of the SD. While determining the SD degradation, the SDSM can be treated as a ratioing radiometer therefore canceling out the on-orbit changes and fluctuations in the gain of SDSM detectors, degradation of the optics as well as any changes in the electronics gain. The SD degradation at SDSM detector $i, \Delta_{i}$, is tracked using the ratios (normalized to the first on-orbit SD/SDSM calibration) of its SD view response $\left(d c_{S D}^{i}\right)$ to its Sun view response $\left(d c_{S U N}^{i}\right)$ as shown in Equation 1. Both the SD and Sun view responses are corrected for the background and the solar illuminating and detector viewing geometries.

$$
\Delta_{i}=\frac{d c_{S D}^{i}}{d c_{S U N}^{i}}
$$

As noted earlier, the SDSM has an attenuation screen permanently in place for the Sun-view. In the case of two MODIS instruments, the transmission of this attenuation screen was not characterized prior to launch. A design error in the SDSM sub-system, not discovered until post-launch, resulted in significantly large ripples appearing in the SDSM Sun view responses of all 9 detectors. To mitigate the impacts of these ripples on the monitoring of the SD degradation, a normalization approach was formulated during the early months of Terra MODIS operation. As the observed ripples exist in all the SDSM detector responses, normalization is made relative to SDSM detector 9 at $0.94 \mu \mathrm{m}$, with the assumption that the degradation at this wavelength is extremely small. ${ }^{5}$ In parallel, a modeling effort to characterize the Sun-view response was also conducted. ${ }^{6}$ As the MODIS missions have continued to operate over a decade beyond their designed life, measurable degradation ( $1 \%$ for Terra MODIS and about $0.5 \%$ for Aqua MODIS) has been observed at $0.94 \mu \mathrm{m}$ (detector 9 ). To account 

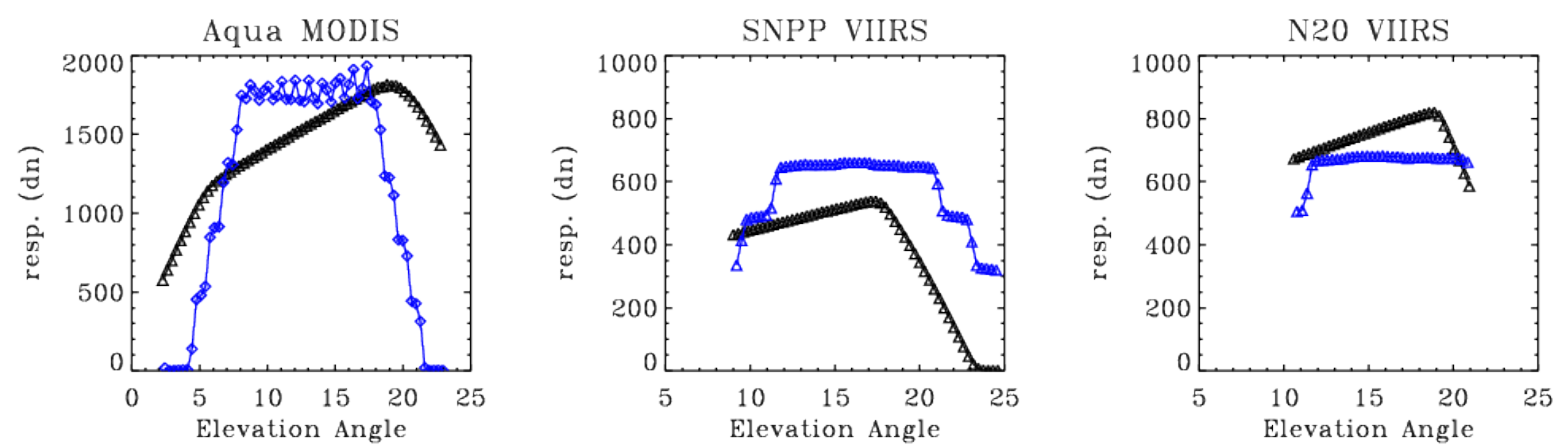

Figure 1. SDSM detector $1(0.412 \mu \mathrm{m})$ response for SD view (black symbols) and Sun-view (blue symbols) for the three instruments from a Jan 2018 calibration events.

for this, an additional correction for the degradation of SDSM detector 9 has already been incorporated in the calibration algorithms for both instruments. ${ }^{7}$

Many lessons from the MODIS design and its on-orbit performance have been incorporated into the VIIRS design and testing. The SDSM Sun-view response screen function was characterized prior to launch and the characterization was improved on-orbit using additional information from the yaw maneuvers. ${ }^{8,9}$ As a result, the SDSM Sun view response for the VIIRS instruments is devoid of the ripples in the signal. The SD degradation in the VIIRS instruments (also referred to as H-factor), includes the transmission functions for both the Sun view screen $\left(\tau_{S U N}\right)$ and the SD view screen $\left(\tau_{S D}\right)$, the initial BRDF of the SD panel $\left(B R D F_{0}\right)$, and a projection correction of the sunlight onto the SD panel $\cos \left(\theta_{\text {inc }}\right)$ and is expressed as ${ }^{10}$

$$
h\left(t_{i}\right)=\frac{1}{N_{i}} \sum_{1}^{N_{i}} \frac{d c_{S D}^{i} \tau_{S U N}}{d c_{S U N}^{i} \tau_{S D} B R D F_{0} \cos \left(\theta_{i n c}\right)}
$$

In the above expression, $N_{i}$ is the number of scans where the SD is fully illuminated. Figure 1 shows the background corrected Sun-view (blue) and SD view (black) responses for SDSM detector $1(0.412 \mu \mathrm{m})$ plotted as a function of elevation angle (degrees). All the trends shown in Figure 1 cover the duration when the SDSM is operating. As noted earlier, the MODIS SDSM operation is a few minutes longer than VIIRS. As discussed earlier, the artifact due to the misalignment of the MODIS Sun-view screen manifests in the form of ripples as seen in Figure 1. A similar behavior is also observed in Terra MODIS. The SDSM D9 normalization approach has worked effectively in mitigating the impact of the observed artifacts and the longevity of the SDSM measurements further reduces the uncertainties associated with the estimation of SD degradation. The effects of the improved Sun-view screen characterization can be observed in both VIIRS instruments that show a very uniform profile of the Sun-view across the elevation angle range of interest. Currently, both VIIRS instruments use the screen characterization function derived from the yaw maneuvers, supplemented with some on-orbit measurements for better coverage of the angular ranges. The difference in the SD response between SNPP and N20 VIIRS is due to the SDs degradation (about $40 \%$ at $0.41 \mu \mathrm{m}$ ) after $6+$ years on-orbit.

The degradation of the SD panel is known to be primarily due to exposure to solar UV light, which has two optical paths to get onto the SD panel: directly via the SD door and scattered light from Earth's atmosphere via the nadir (Earth view port). In the case of the two MODIS instruments, the scattered light from the nadirport is the dominant source of degradation due to the design of the scan mirror. On the contrary, the rotating telescope assembly (RTA) in the VIIRS instruments has improved baffling therefore minimizing the effects of the scattering via the Earth view port. As the four instruments are operating on different timescales, the optimal way to compare the SD degradation is to model it as a function of the solar exposure time. The gain degradation of the SDSM detectors can be evaluated using the time-series of Sun-view measurements for each individual detector after applying the necessary corrections. A comparison of the SDSM detector gain change on-orbit is performed for the four instruments. 


\section{RESULTS AND DISCUSSIONS}

As the four instruments have varying operational timelines and configurations, an evaluation of the SD's degradation needs to be performed carefully. The results are divided into two timelines: a) the first 180 days of N20 VIIRS on-orbit performance compared with SNPP VIIRS and Aqua MODIS and b) the first six years on-orbit performance of SNPP VIIRS compared with the two MODIS instruments. For each instrument (SDSM detector), the SD degradation versus solar exposure (hours) is calculated independently. Figure 2a shows the SD degradation for SDSM detector 1 (of both MODIS and VIIRS instruments) plotted as a function of solar exposure. While Aqua MODIS and SNPP VIIRS have both experienced greater than 30 hours of solar exposure, the plot below has been truncated to compare with N20 VIIRS. Aqua MODIS has least degradation during the initial few years as the SD door is only opened during a scheduled SD calibration. On the contrary, SNPP VIIRS does not have a SD door and as a result experiences more degradation. As of June 20, 2018, N20 VIIRS SD has degraded up to $9 \%$ at the $0.41 \mu \mathrm{m}$ wavelength, about $3 \%$ lower than SNPP on a similar timescale. Figure $2 \mathrm{c}$ shows similar results for the $0.74 \mu \mathrm{m}$ detector that shows very minimal $(<1 \%)$ degradation for the VIIRS instruments and even smaller for Aqua MODIS. Although the SD degradation for MODIS is derived using the normalization approach, some residual oscillations still exist in the SD degradation trend across all detectors. As discussed earlier, the improved SDSM screen alignment in the VIIRS instruments has manifested into a smoother time-series of SD degradation measurements as compared to MODIS. The SNPP and N20 VIIRS SD screen characterization has been performed using a combination of measurements from the yaw-maneuvers, supplemented by regular SD calibration events to provide a better angular coverage. In Figures $2 \mathrm{~b}$ and $2 \mathrm{~d}$, similar results for SNPP VIIRS and MODIS instruments are plotted corresponding to the first 250 hours of solar exposure (over the lifetime of SNPP operations). As expected, Terra MODIS that now operates in an SNPP-like configuration has more solar exposure, accentuated after the SD door anomaly of 2003, after which it effectively operates like a VIIRS instrument with solar exposure from the SD door every orbit. In general, a consistent trend of more SD degradation at short-wavelengths, albeit of varying magnitudes, is observed across the four instruments.

In Figure 3, the cumulative degradation of the SD for the MODIS and VIIRS instruments is shown. In the first six months on-orbit, it is clear that SNPP VIIRS SD has experienced most degradation at wavelengths less than $0.6 \mu \mathrm{m}$. At wavelengths greater than $0.6 \mu \mathrm{m}$, a consistent behavior is observed between the two VIIRS instruments. Over a longer-time scale, Terra MODIS has experienced the most degradation, as seen in Figure $3 \mathrm{~b}$, primarily due to the prolonged solar exposure after the SD door anomaly. SNPP VIIRS, operating in a similar configuration, also exhibits over $40 \%$ degradation (at $0.41 \mu \mathrm{m}$ ) of its SD after six years on-orbit and N20 VIIRS SD is also expected to follow a similar pattern. The SDSM detector's wavelength coverage restricts it from tracking the SD's degradation at SWIR wavelengths. On-orbit lunar measurements as well as Earth-view measurements for both instruments have confirmed up to $1 \%$ degradation of the SD at $1.2 \mu \mathrm{m}$ (with Terra MODIS SD showing the largest degradation) and less than $1 \%$ for longer SWIR wavelengths. Consequently, an empirical model based on the SDSM measurements is used for the MODIS and SNPP VIIRS instruments to track the SD's degradation at SWIR wavelengths. ${ }^{11,12}$ A similar approach is also planned for N20 VIIRS in the future.

One of the other important metrics that needs to be monitored is the performance of the SDSM's detectors. Although the SDSM acts as a ratioing radiometer to effectively cancel out the effect of on-orbit detector gain changes on the estimated SD degradation, long-term performance monitoring of the SDSM detectors is warranted. Due to its degradation, the SD cannot provide an accurate gain change of the SDSM detector, especially at short wavelengths. Therefore, the normalized Sun-view response, corrected for background, view geometry parameters, and earth-sun distance is used to estimate the long-term gain change of the SDSM detectors. In the case of MODIS, the artifacts due to the SDSM Sun-screen misalignment add additional uncertainty to the trending; however, the longevity of the MODIS missions facilitates a reasonable estimation of gain of the SDSM detectors. Figure 4a shows the normalized gain (Sun response trending) at $0.41 \mu \mathrm{m}$ for the two VIIRS instruments and Aqua MODIS for the first 200 days. Figure 4c shows a similar trending for the last SDSM detector (D9 for MODIS and D8 for VIIRS at $0.93 \mu \mathrm{m}$ ). Previous work has documented that the most gain degradation for the SDSM detectors occurs at the longer wavelengths due to high energy protons. ${ }^{13,14}$ The normalized gain trends for the D1 and D8 shown in Figures 4a and 4c reaffirm this previous observation where the N20 VIIRS, with a similar SDSM design follows closely with the SNPP VIIRS for both detectors. A maximum of $18 \%$ change in 


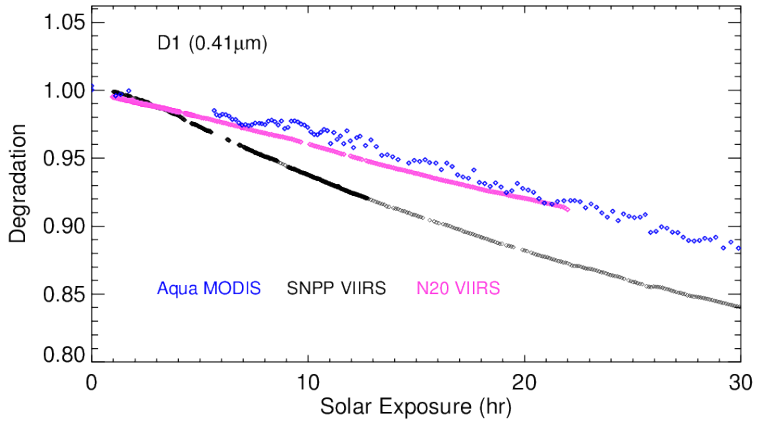

(a)

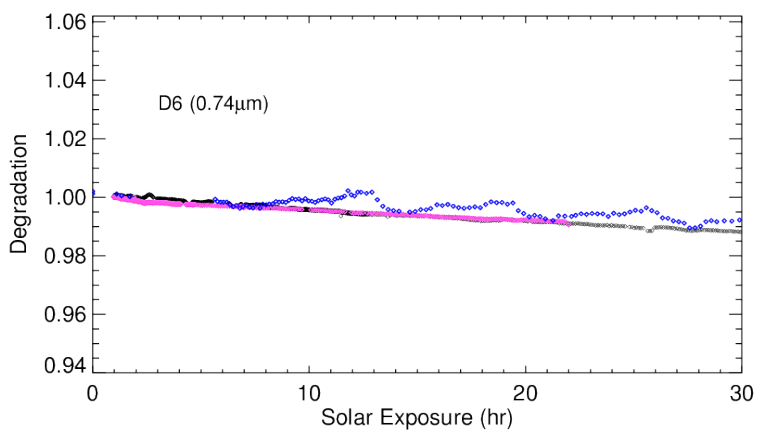

(c)

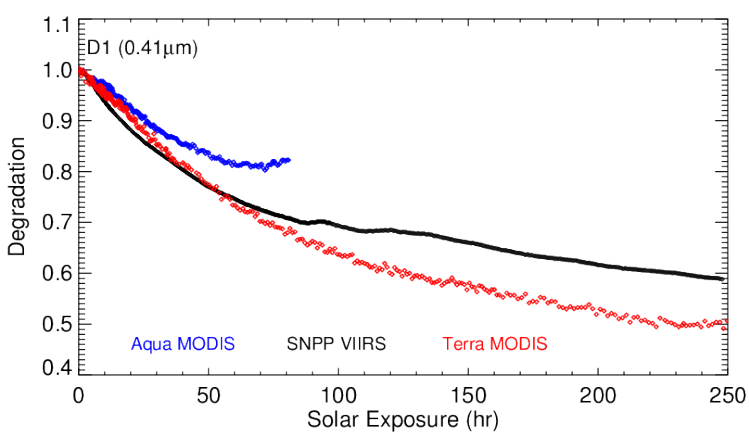

(b)

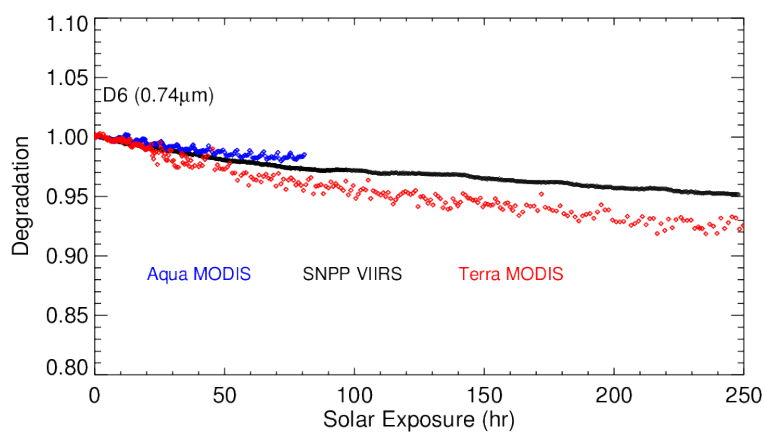

(d)

Figure 2. SD degradation for the MODIS and VIIRS instruments as a function of Solar Exposure (hours). Aqua MODIS, SNPP and N20 VIIRS compared on a multi-month timescale and Terra and Aqua MODIS compared with SNPP on a multi-year time scale. (a) Initial SD degradation comparison at $0.412 \mu \mathrm{m}$ (SDSM D1) (b) Mission-long SD degradation comparison at $0.412 \mu \mathrm{m}$ (c) Initial SD degradation comparison at $0.74 \mu \mathrm{m}$ (SDSM D6) (d) Mission-long SD degradation comparison at $0.74 \mu \mathrm{m}$ (SDSM D6)

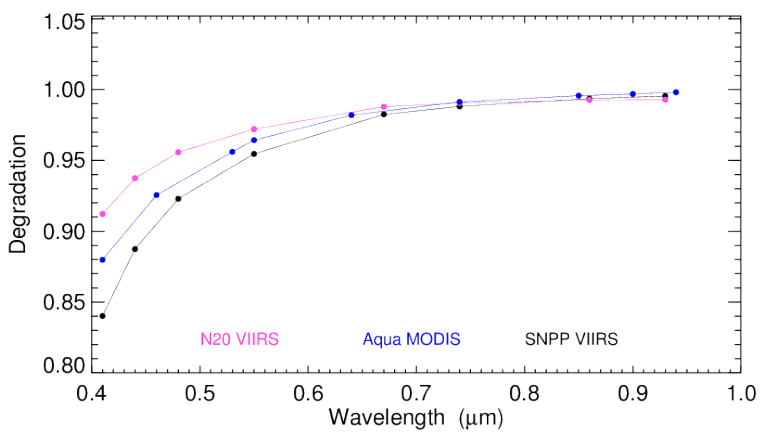

(a)

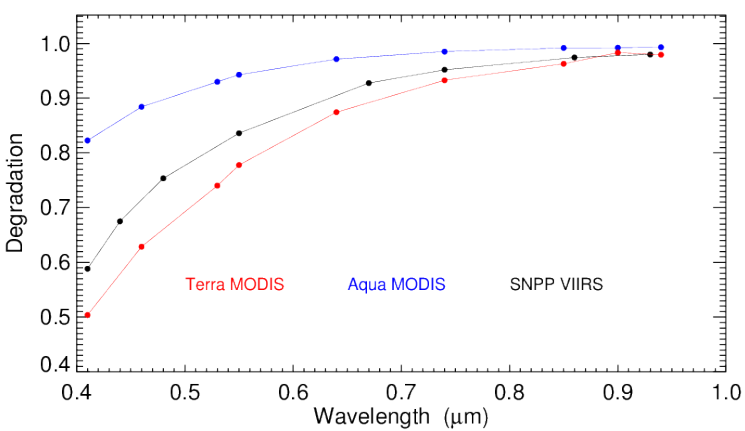

(b)

Figure 3. (a) Cumulative SD degradation for the MODIS and VIIRS instruments after 30 hours of solar exposure on-orbit (22 hours for N20 VIIRS). (b) Cumulative SD degradation for the MODIS and VIIRS instruments after 250 hours of solar exposure on-orbit 


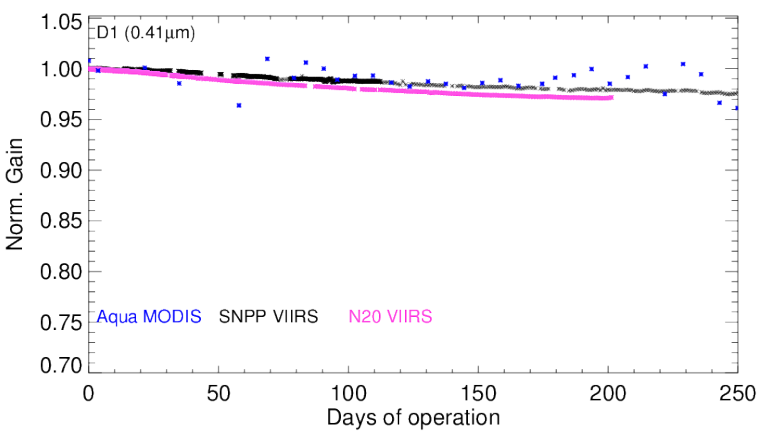

(a)

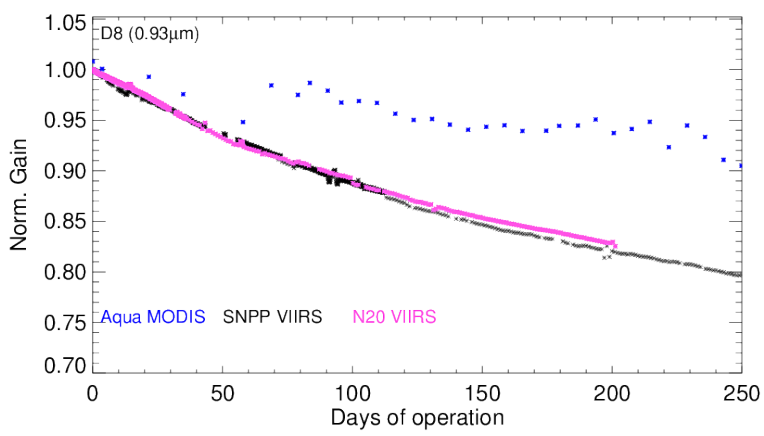

(c)

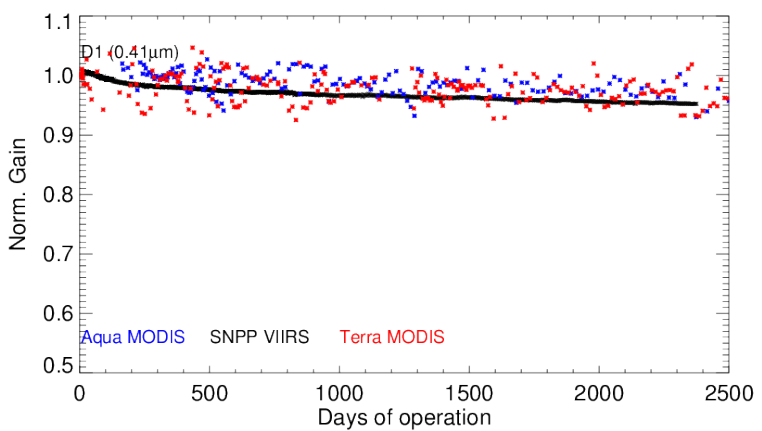

(b)

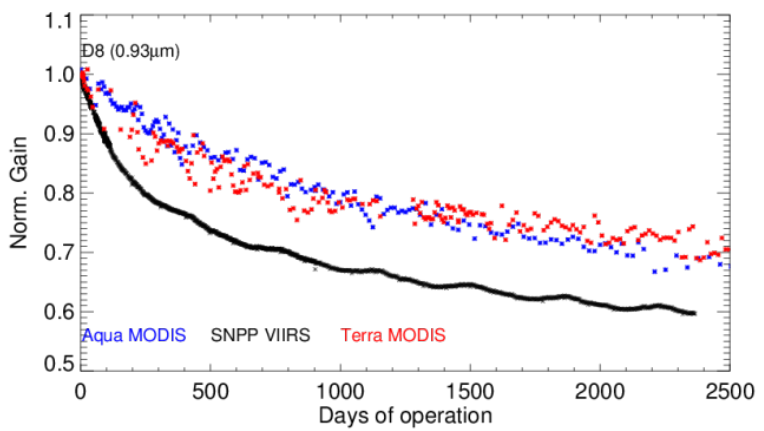

(d)

Figure 4. Normalized Gain of SDSM detectors. Aqua MODIS, SNPP and N20 VIIRS compared on a multi-month timescale and Terra and Aqua MODIS compared with SNPP on a multi-year time scale. (a) SDSM gain trending at $0.412 \mu \mathrm{m}$ (SDSM D1) (b) Mission-long SDSM gain trending comparison at $0.412 \mu \mathrm{m}$ (c) SDSM gain trending at $0.93 \mu \mathrm{m}$ (SDSM D8) (d) SDSM gain trending at $0.93 \mu \mathrm{m}$ (SDSM D8)

the gain is observed for SNPP after 180 days on-orbit with N20 VIIRS gain also projected to change by a similar magnitude. In contrast, Aqua MODIS SDSM detectors did not show significant gain change in the first 180 days on-orbit. In Figures $4 \mathrm{~b}$ and 4d, a multi-year gain trend for the detectors 1 and 8 of SNPP VIIRS and the corresponding detectors (D1 and D9) of MODIS are plotted. In order to compare the trend with SNPP, only the first six years of Terra and Aqua MODIS data is presented. While the two MODIS instruments show reasonable agreement in terms of gain change after 6 years on-orbit, the normalized gain for SNPP VIIRS SDSM D8 has experienced up to $10 \%$ more degradation. Figures $5 \mathrm{a}$ and $5 \mathrm{~b}$ show the cumulative gain change across all SDSM detectors after 180 days and 6 years on-orbit respectively. All four instruments exhibit a consistent trend with the largest gain change at NIR wavelengths. Radiation testing was performed prior to launch at three levels and the degradation at $0.9 \mu \mathrm{m}$ was determined to be similar to that observed on-orbit. With SNPP's (and N20) orbit $(824 \mathrm{~km})$ higher than Aqua and Terra $(705 \mathrm{~km})$, the radiation environment is known to be roughly $2 \mathrm{x}$ higher than the Aqua and Terra orbits for proton flux. This is a likely explanation for the large gain change in SDSM detectors in VIIRS as compared to MODIS. ${ }^{14}$

\section{SUMMARY}

A brief overview of the MODIS and VIIRS SDSM design, their on-orbit operation and calibration strategies is presented in this paper. The comparison of the SNPP and N20 VIIRS on-orbit performance, in terms of changes in SD BRF and SDSM detector responses, with the two MODIS instruments is presented. A common behavior of more SD degradation at shorter wavelengths is observed across the four instruments. Due to its operational configuration, Aqua MODIS SD experiences lower solar exposure compared to SNPP VIIRS and Terra MODIS. 


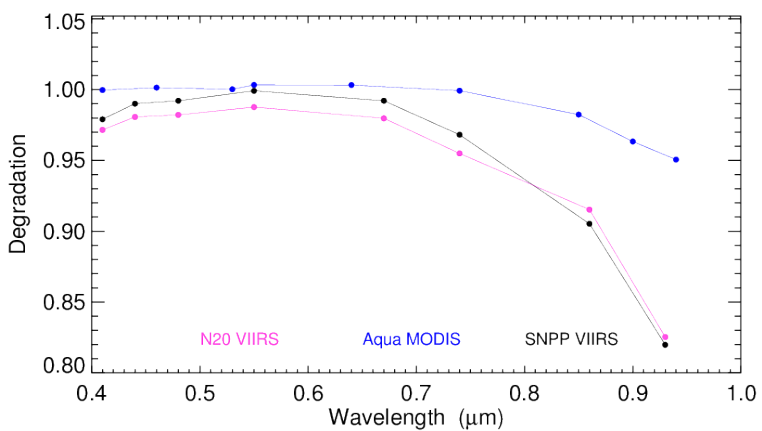

(a)

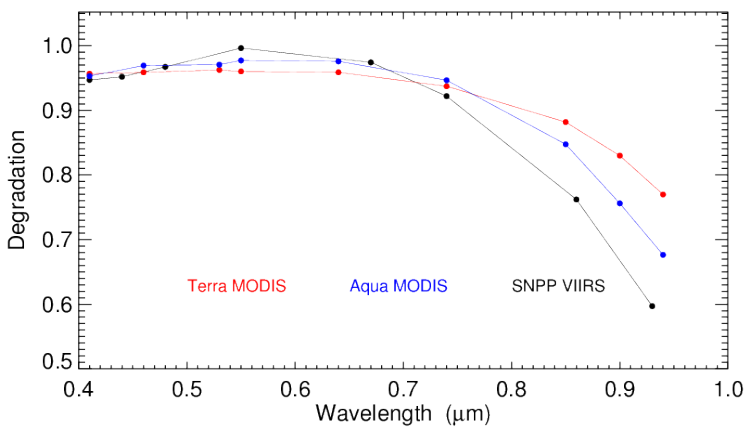

(b)

Figure 5. (a) Cumulative SDSM gain change for the MODIS and VIIRS instruments after 180 days on-orbit. (b) Cumulative SDSM gain change for the Terra and Aqua MODIS and SNPP VIIRS instruments after 6 years on-orbit

As a result, the observed degradation of the SD is also smaller compared to SNPP and Terra MODIS. The MODIS and VIIRS SDSM detectors are from the same manufacturer and also show similar behavior on-orbit. The normalized gain, computed using the Sun-view response, for each instrument reveals long-term response degradation for the NIR detectors, with most degradation observed in SNPP VIIRS.

\section{ACKNOWLEDGMENTS}

Authors in this paper would like to acknowledge the efforts of the MODIS and VIIRS instrument vendor (Raytheon) and other MCST and VCST members (previous and current). The authors would also like to thank Dr. Tiejun Chang, MCST for technical review of this manuscript.

\section{REFERENCES}

[1] Barnes, W. L., Pagano, T. S., and Salomonson, V. V., "Prelaunch characteristics of the Moderate Resolution Imaging Spectroradiometer (MODIS) on EOS-AM1," IEEE Transactions on Geoscience and Remote Sensing 36(4), 1088-1100 (1998).

[2] Murphy, R., Ardanuy, P., Deluccia, F. J., Clement, J., and Schueler, C. F., "The Visible Infrared Imaging Radiometer Suite," in [Earth Science Satellite Remote Sensing], 199-223, Springer (2006).

[3] Xiong, X. J., Angal, A., Sun, J., Choi, T. J., and Johnson, E., "On-orbit performance of MODIS solar diffuser stability monitor," Journal of Applied Remote Sensing 8(1), 083514 (2014).

[4] Xiong, X., Fulbright, J., Angal, A., Wang, Z., Geng, X., and Butler, J., "Assessment of MODIS and VIIRS solar diffuser on-orbit degradation," in [Earth Observing Systems XX], 9607, 96071T, International Society for Optics and Photonics (2015).

[5] Xiong, X., Esposito, J. A., Sun, J.-Q., Pan, C., Guenther, B. W., and Barnes, W. L., "Degradation of MODIS optics and its reflective solar bands calibration," in [Sensors, Systems, and Next-Generation Satellites V], 4540, 62-71, International Society for Optics and Photonics (2001).

[6] Sun, J.-Q., Xiong, X., and Barnes, W. L., "MODIS solar diffuser stability monitor sun view modeling," IEEE Transactions on Geoscience and Remote Sensing 43(8), 1845-1854 (2005).

[7] Chen, H., Xiong, X., Angal, A., and Twedt, K. A., "On-orbit Characterization of the MODIS SDSM Screen for Solar Diffuser Degradation Estimation," IEEE Transactions on Geoscience and Remote Sensing 55(11), 6456-6467 (2017).

[8] McIntire, J., Moyer, D., Efremova, B., Oudrari, H., and Xiong, X., "On-orbit Characterization of S-NPP VIIRS Transmission Functions," IEEE Transactions On Geoscience And Remote Sensing 53(5), 2354-2365 (2015).

[9] Lei, N., Chen, X., and Xiong, X., "Determination of the SNPP VIIRS SDSM Screen Relative Transmittance From Both Yaw Maneuver and Regular On-Orbit Data," IEEE Transactions On Geoscience And Remote Sensing 54(3), 1390-1398 (2016). 
[10] Fulbright, J., Lei, N., Efremova, B., and Xiong, X., "Suomi-NPP VIIRS Solar Diffuser Stability Monitor Performance," IEEE Transactions On Geoscience And Remote Sensing 54(2), 631-639 (2016).

[11] Twedt, K. A., Angal, A., Xiong, X., Geng, X., and Chen, H., "MODIS solar diffuser degradation at short-wave infrared band wavelengths," in [Earth Observing Systems XXII], 10402, 104022K, International Society for Optics and Photonics (2017).

[12] Lei, N. and Xiong, X., "Suomi NPP VIIRS Solar Diffuser BRDF Degradation Factor at Short-Wave Infrared Band Wavelengths," IEEE Transactions on Geoscience and Remote Sensing 54(10), 6212-6216 (2016).

[13] De Luccia, F., Moyer, D., Johnson, E., Rausch, K., Lei, N., Chiang, K., Xiong, X., Fulbright, J., Haas, E., and Iona, G., "Discovery and characterization of on-orbit degradation of the Visible Infrared Imaging Radiometer Suite (VIIRS) Rotating Telescope Assembly (RTA)," in [Earth Observing Systems XVII], 8510, 85101A, International Society for Optics and Photonics (2012).

[14] Iona, G., Butler, J., Guenther, B., Graziani, L., Johnson, E., Kennedy, B., Kent, C., Lambeck, R., Waluschka, E., and Xiong, X., "VIIRS on-orbit optical anomaly: Investigation, analysis, root cause determination and lessons learned," in [Earth Observing Systems XVII], 8510, 85101C, International Society for Optics and Photonics (2012). 